\title{
3D SIMULATION STUDY OF INHOMOGENEOUS MICROSTRUCTURE AND ITS EVOLUTION (advantages of visual simulation technique in stereological analysis)
}

\author{
XiAOYAN SONG ${ }^{1}$, MARKUS RETTENMAYR ${ }^{2}$ AND GUOQUAN LiU ${ }^{3}$ \\ ${ }^{1}$ School of Materials Science and Engineering, Beijing University of Technology, 100022 Beijing, China, \\ ${ }^{2}$ Materials Science Institute, Darmstadt University of Technology, 64287 Darmstadt, Germany, ${ }^{3}$ School of \\ Materials Science and Engineering, University of Science and Technology Beijing, 100083 Beijing, China \\ e-mail: xysong@bjpu.edu.cn \\ (Accepted October 15, 2003)
}

\begin{abstract}
A 3D Monte Carlo technique has been used to simulate a complex process of recrystallization in a material containing precipitates after graded deformation. By the simulation model, the inhomogeneous deformed microstructure and its evolution during subsequent annealing were directly displayed; moreover, the locally varying recrystallized microstructure was quantitatively analyzed. The simulation results were well verified by experiments with an aluminum alloy containing $\mathrm{Al}_{3} \mathrm{Zr}$ particles that had been subjected to deformation and annealing for recrystallization. In the stereological analysis of a non-random microstructure and its evolution kinetics, the visual simulation technique showed apparent advantages for sampling, detection and measuring.
\end{abstract}

Keywords: 3D computer visualization, inhomogeneous microstructure, Monte Carlo technique, recrystallization, stereology.

\section{INTRODUCTION}

Computer visualization of microstructure has been rapidly developed during the past decade in the field of materials science and engineering since Srolovitz et al. (1983) for the first time used a 2D Monte Carlo method to simulate normal grain growth. Nowadays, image simulation techniques, mainly including Monte Carlo method (Rollett et al., 1992; Song et al., 2001), cellular automata (Raabe, 2002), vertex model (Enomoto and Kato, 1990) and phase-field theory (Krill and Chen, 2002), have been widely applied to simulate various processes found in materials research \& development.

Recrystallization, as a common phenomenon that is observed in most of the metallic materials after plastic deformation and annealing, has been studied with computer modeling by some researchers (see overview by Doherty et al. (1997)). A major part of this modeling work has been concentrating on fundamental processes, e.g. recrystallization in singlephase materials, assuming uniform deformation that leads to homogeneous microstructures. However, the uniform deformation cannot be actually attained on the length scale of the sample or even on the length scale of the grain. Therefore, inhomogeneous recrystallization is very often encountered in industry, especially in materials containing fine particles as a second reinforcing phase.

In the present work, a 3D Monte Carlo technique is developed to model recrystallization after inhomogeneous deformation in a material containing particles. In the complex case where microstructure reveals large scale inhomogeneity and evolves with time, it is difficult and under some circumstances it is impossible to characterize the microstructural features with the conventional stereological methods. It will be shown that, in such cases, 3D visual simulation offers advantages both morphologically and quantitatively.

\section{GRADED DEFORMATION}

An Al-0.5wt.\%Zr alloy was used in the experiments, containing the dispersed $\mathrm{Al}_{3} \mathrm{Zr}$ particles that had formed after precipitation heat treatment at $480^{\circ} \mathrm{C}$ for $24 \mathrm{hrs}$. The mean diameter $d_{p}=200 \mathrm{~nm}$ and volume fraction $f_{p}=0.04$ of the randomly distributed precipitates were measured with conventional stereological techniques (Exner and Hougardy, 1988; $\mathrm{Yu}$ and Liu, 1989) on high resolution scanning microscopy (HRSEM) images. 
To study microstructure evolution under the condition of inhomogeneous deformation, a graded deformation was introduced into the sample. A special shape with varying initial height had been designed for the sample which was subjected to subsequent deformation by means of cold rolling. The typical shapes together with the sizes of the sample before and after deformation are shown in Fig. 1. Thus, the rolling reduction (defined as the relative decrease of the height) can be calculated, varying from $60 \%$ to $90 \%$ along the longitudinal direction of the deformed sample. A number of equidistant straight lines perpendicular to the longitudinal direction were marked on the top surface of the graded part of the undeformed sample (see Fig. 1a) to determine the function of the deformation gradient. Under conditions of volume conservation and a constant width of the sample during deformation process due to cold rolling, the following relationship should hold,

$$
\Delta x_{i+1}^{d}-\Delta x_{i}^{d}=\frac{1}{h^{d}}\left(\Delta x^{u}\right)^{2} \sin \theta \cos \theta,
$$

where $\Delta x^{u}$ is the initial distance before deformation between equidistant lines, $\Delta x_{i}^{d}$ is a distance measured between two marked lines after deformation, $h^{d}$ is the constant height of sample after deformation, and $\theta$ is the angle of inclination of the graded part of the undeformed sample to the horizontal plane. The right side of Eq. 1 consists only of constants, indicating that after deformation the increase of the distances between the lines is constant. This is approximately confirmed by corresponding measurements on the deformed sample (Fig.1b). Therefore, in the part containing the deformation gradient, the rolling reduction can be characterized as a linearly increasing function of the distance along the rolling direction.

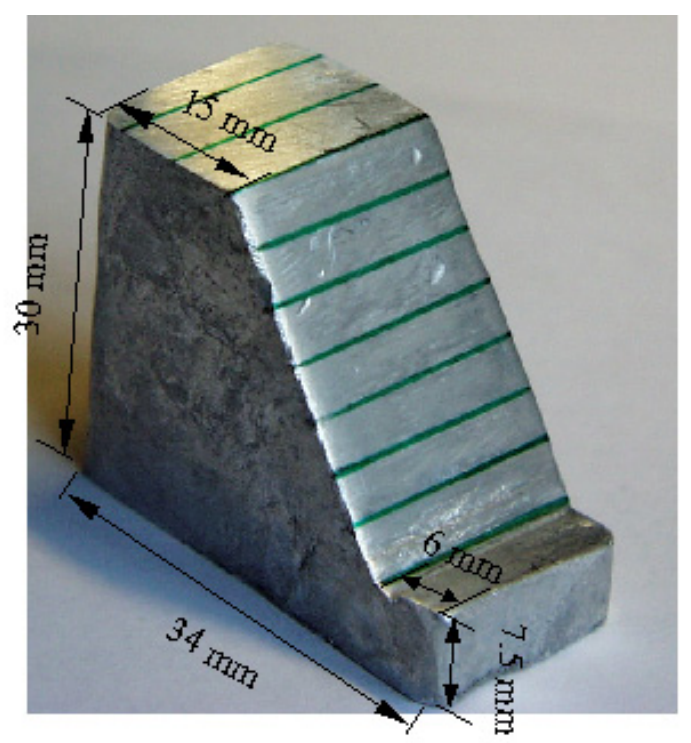

a)

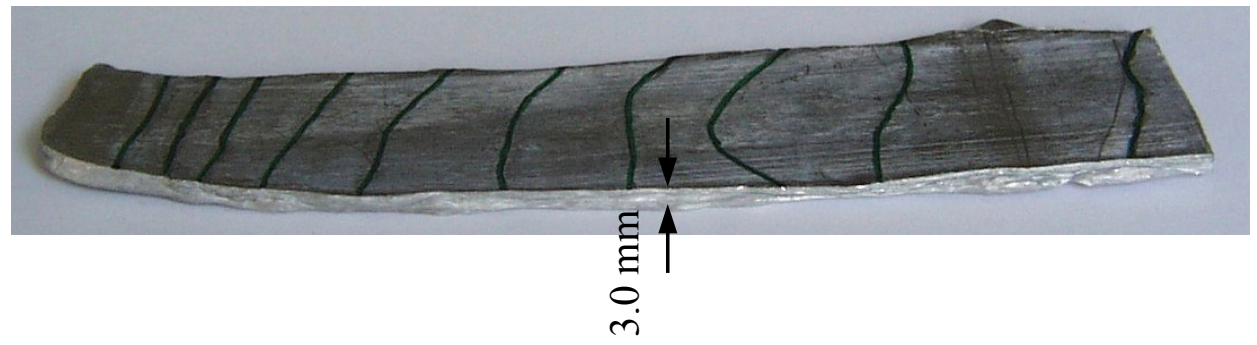

b)

Fig. 1. Pictures of sample before a) and after b) deformation by cold rolling. 


\section{SIMULATION MODEL}

At first, the initial microstructure with equiaxed grains is generated with a Monte Carlo algorithm for normal grain growth (Song and Liu, 1998). The fine precipitates are distributed randomly in the matrix, which is represented by a cubic micro-unit structure of uniform size. The deformed grain structure is then generated by tracing the positions of micro-units which belong to the same grain before deformation and whose positions are changed according to the local rolling reduction. As a result of locally varying reductions, the deformed grains at the transition zone from lower deformation to higher deformation will have an upward macroscopic curvature. This is demonstrated by the shape change of a circular zone representative of an ideal equiaxed grain, as shown in Fig. 2. The geometry of the simulated domain with a shape equivalent to that of the experimental sample is shown in Fig. 3a, with the corresponding deformed microstructure in Fig. 3b. To restrict computing time, only a part $(1113 \times 30 \times 100$ cubic units $)$ of the total volume $(2463 \times 30 \times 100$ cubic units) is used that contains the deformation gradient plus parts of the adjacent zones with approximately uniform deformation.

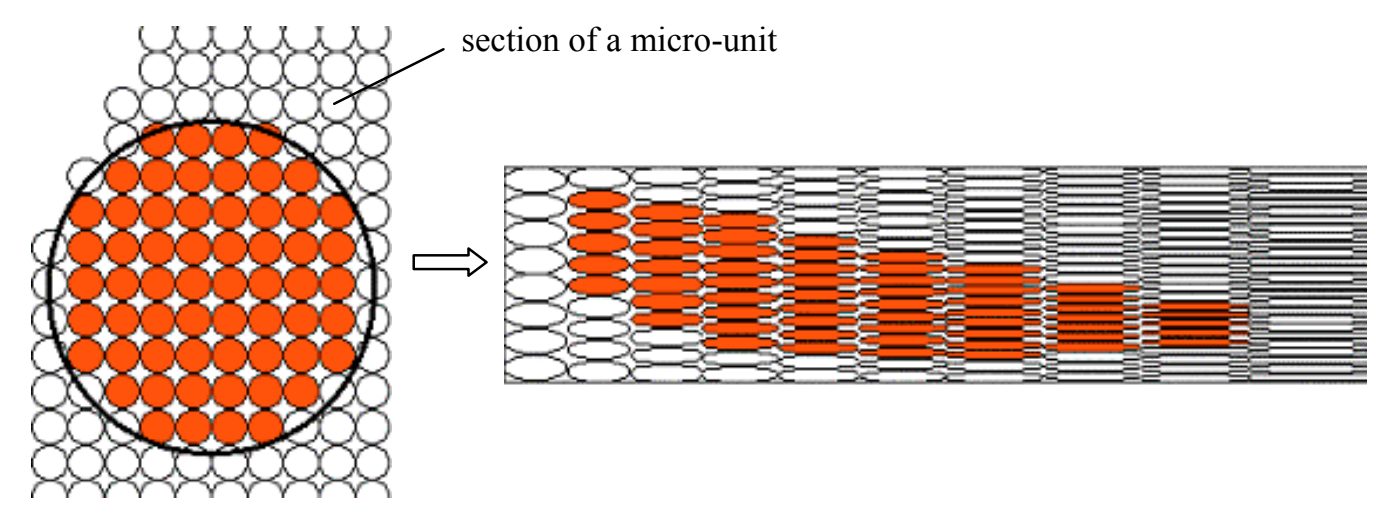

Fig. 2. Demonstration of deformed geometry due to cold rolling of a circular zone, representing an equiaxed grain; the reduction varies from $25 \%$ to $80 \%$ at the left and right borders respectively.

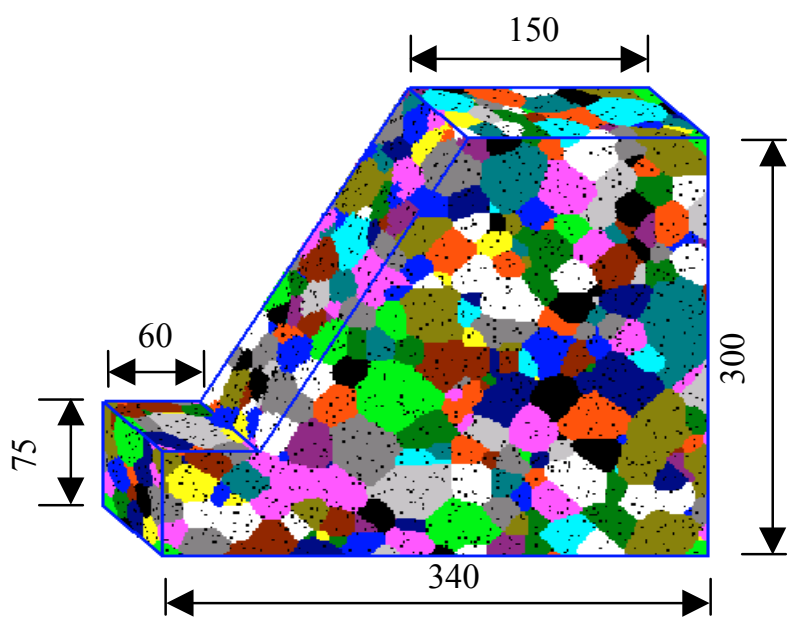

a)

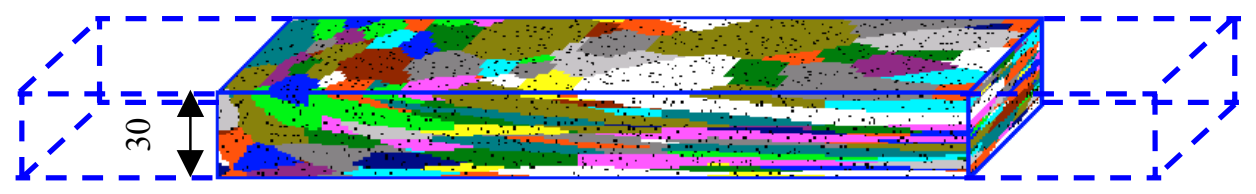

b)

Fig. 3. Simulated 3D microstructures before a) and after b) deformation; both geometries are equivalent to those of the experimental sample. Dimensions are given as numbers of Monte Carlo micro-units. 
Next, the local stored energy that is calculated from the local strain is mapped onto the micro-units that build the deformed microstructure. Nucleation of recrystallization is assumed to occur instantaneously at positions where the local stored energy $E_{D}(x)$ is higher than the sum of the critical stored energy for recrystallization $E_{D}^{C}$ and the pinning force from the precipitates $F_{p}$. The local nucleation rate $\dot{N}(x)$ can therefore be expressed as

$$
\dot{N}(x)=C_{0}\left(E_{D}(x)-E_{D}^{C}-F_{p}\right) \exp \left(\frac{-Q}{R T}\right) .
$$

$Q$ is the activation energy of nucleation, $R$ is the gas constant, and $T$ is the absolute temperature. $C_{0}$ is the rate constant that is used here as a fitting parameter. During the first simulation time unit (Monte Carlo Step, MCS), the local nucleus density is calculated with Eq. 2. Initially, the nuclei are given the uniform size of the micro-unit.

The microstructure evolution with time is then simulated by tracking the re-orientation tendencies of each micro-unit with respect to the orientation state of one of its randomly selected neighbors. Microunits that represent precipitates are excluded from selection as reorienting or neighboring units. The local energy $E_{t}$ at time $t$ of a micro-unit is determined using the formalism

$$
E_{t}=g\left(S_{i}\right) E_{D}(x)+\sum_{K=1}^{N_{K}} \sum_{j \in K} J\left(1-\delta_{S_{i} S_{j}}\right),
$$

where $S_{i}$ is the orientation index of unit $i$, and $g\left(S_{i}\right)$ is a step function of $S_{i} \cdot g\left(S_{i}\right)$ equals unity or zero when unit $i$ is an unrecrystallized or a recrystallized micro-unit, respectively. The second term on the right side of Eq. 3 represents the local interface energy (Song and Liu, 1998). At time $t+\Delta t$, when unit $i$ strives to reorient to a new orientation with index as $S_{i}^{\prime}$, the tentative new energy state of unit $i$ is calculated by

$$
E_{t+\Delta t}=\sum_{K=1}^{N} \sum_{j \in K} J\left(1-\delta_{S_{i}^{\prime} S_{j}}\right)+F_{p} .
$$

The reorientation probability depends on the change of the energy $\Delta E=E_{t+\Delta t}-E_{t}$. To characterize the varying migration velocities of the recrystallizing grain boundaries due to the graded stored energy, the relationship between the reorientation probability and the energy change is determined quantitatively. When the new energy state according to Eq. 4 is lower than the original energy state (Eq. 3), the orientation change is effectuated with a probability that corresponds to the ratio between the absolute value of $\Delta E$ and the maximum possible decrease of energy when the highest local stored energy is released. If the new energy state is exceeding the old one, the former orientation is maintained.

\section{RESULTS}

The simulated recrystallizing microstructures can be displayed in 3D at any simulation step. Hence, the microstructure evolution during recrystallization can be directly observed. Two simulated 3D microstructures representing an intermediate state and the stabilized recrystallization state are shown in Fig. $4 a$ and $4 b$, respectively. The recrystallized grains can be identified in the deformed matrix with elongated grains by the approximately equiaxed shape. The two-dimensional microstructures in sections including the rolling direction and the direction normal to the rolling surface (i.e. those parallel to XOY plane, as indicated in Fig. 4) are microstructures generally investigated in metallography. Additionally, microstructures in sections parallel to the rolling surface (i.e. those parallel to XOZ plane in Fig. 4) and perpendicular to the rolling direction (i.e. those parallel to $\mathrm{YOZ}$ plane in Fig. 4) are also displayed.

The three-dimensional perspective effect of simulation provides an appropriate and efficient way to study inhomogeneous microstructures as well as their evolution. The gradually increasing nucleus density with distance from the end of the lowest deformation state is reflected by the microstructure at an early stage of recrystallization, and also by the distribution of recrystallizing grains at intermediate stages (Fig. 4a). The locally varying grain growth velocities result in different increases in the size of the recrystallizing grains at different locations after the same time interval, as shown by a sequence of microstructures revealed by YOZ sections (Fig. 4a). After long simulation times, the local (and, hence, the total) recrystallized volume fraction remains essentially constant as a result of the energy balance between the stored energy, acting as the driving force for nucleation and the growth of recrystallizing grains, and the dragging force from the fine particles. Consequently, a final stabilized microstructure with the graded sizes and volume fractions of the recrystallized grains is obtained (Fig. 4b). 
Experiments were carried out for validation purpose. The deformed sample shown in Fig. $1 \mathrm{~b}$ was at $380^{\circ} \mathrm{C}$ during 3 hours annealed for recrystallization. Afterwards, a series of parts of the sample were prepared metallurgically for the optical microscope and the scanning electron microscope (SEM). The inhomogeneously recrystallized microstructure was observed. Many stereological techniques that require random sampling for generating unbiased estimates $(\mathrm{Yu}$ and Liu, 1989) cannot be applied to microstructures with non-randomly distributed features. To describe as completely as possible the characteristics of such an inhomogeneous microstructure, 90 pictures were taken of sections that included the rolling direction and the direction normal to the rolling surface of the sample. As an example, Fig. 5 depicts a transition zone with respect to the deformation gradient. The macroscopic curvature of the deformed grains, the lower nucleus density and the smaller sizes of recry- stallizing grains in the zones closer to the low deformation end, are in qualitative agreement with the simulation predictions (see Fig. 4). The recrystallized volume fractions as a function of position, obtained separately from experiments and simulations, are compared in Fig. 6. Good quantitative agreement between the experimental and simulation results is found.

It is worth pointing out that, a traditional stereological technique as serial sectioning (Exner and Hougardy, 1988; Yu and Liu, 1989) perpendicular to the rolling direction (referring to Fig. 4a), would be more precise for characterizing the inhomogeneous microstructure in experiments. However, it will be enormously time- and energy-consuming (thus, impractical) to acquire the microstructural features of the whole sample with sufficient accuracy. Thus, in this respect, the visual simulation technique is obviously superior to the experimental methods.

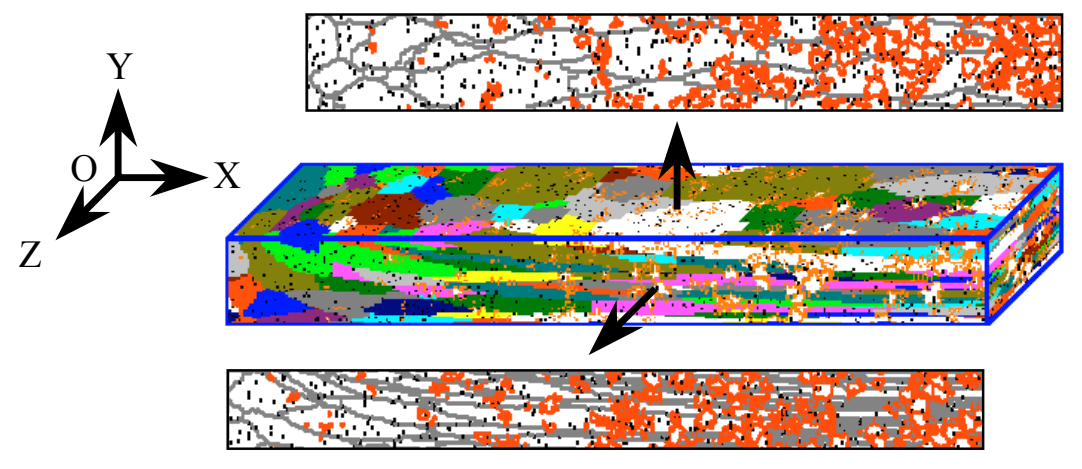

sections parallel to YOZ plane:
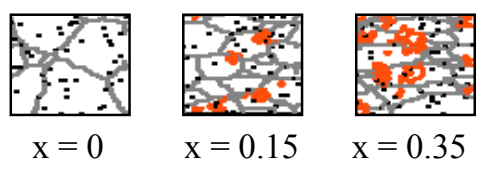

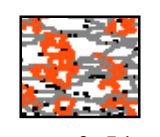

$\mathrm{x}=0.51$

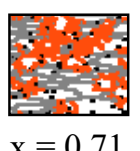

$\mathrm{x}=0.71$

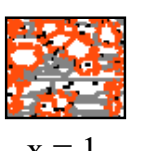

$\mathrm{x}=1$

a)

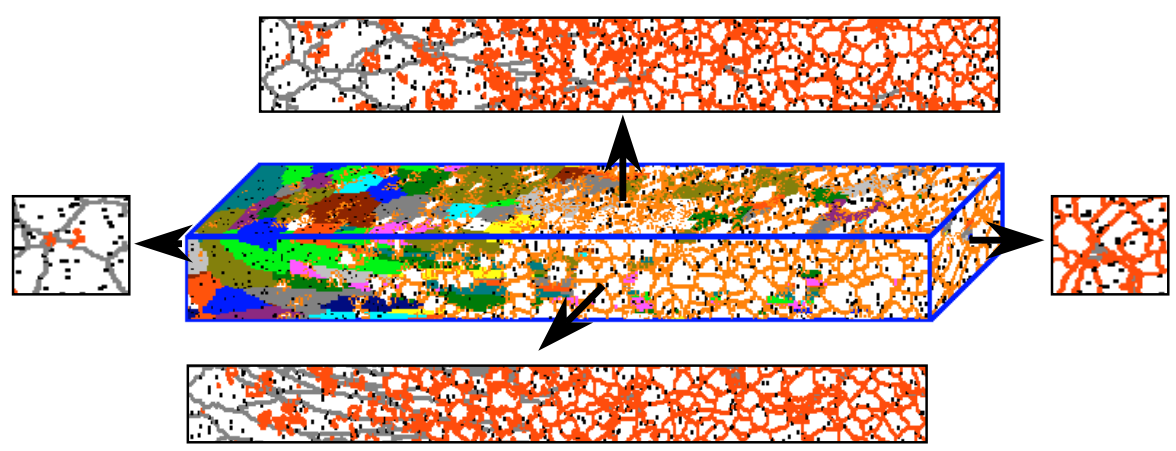

b)

Fig. 4. Simulated microstructure evolution during recrystallization: a) intermediate state, with $x$ as the relative distance from the low deformation end; b) final stabilized state. 


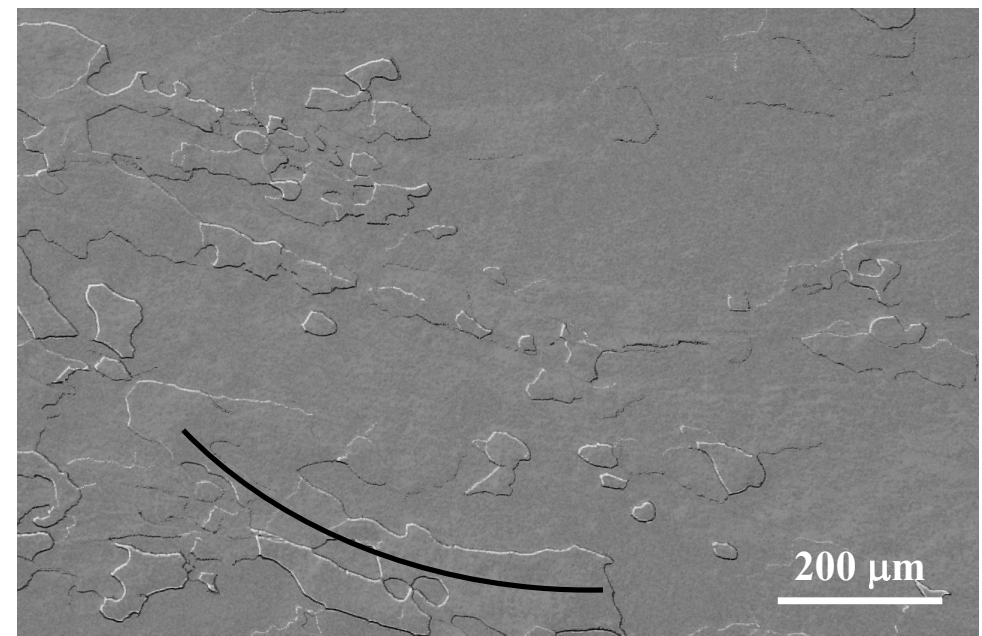

Fig. 5. Partially recrystallized microstructures of Al-0.5\%Zr sample, taken at a relative distance $x=0.05$ from the low deformation end. The line indicates the curved shape of deformed grains.

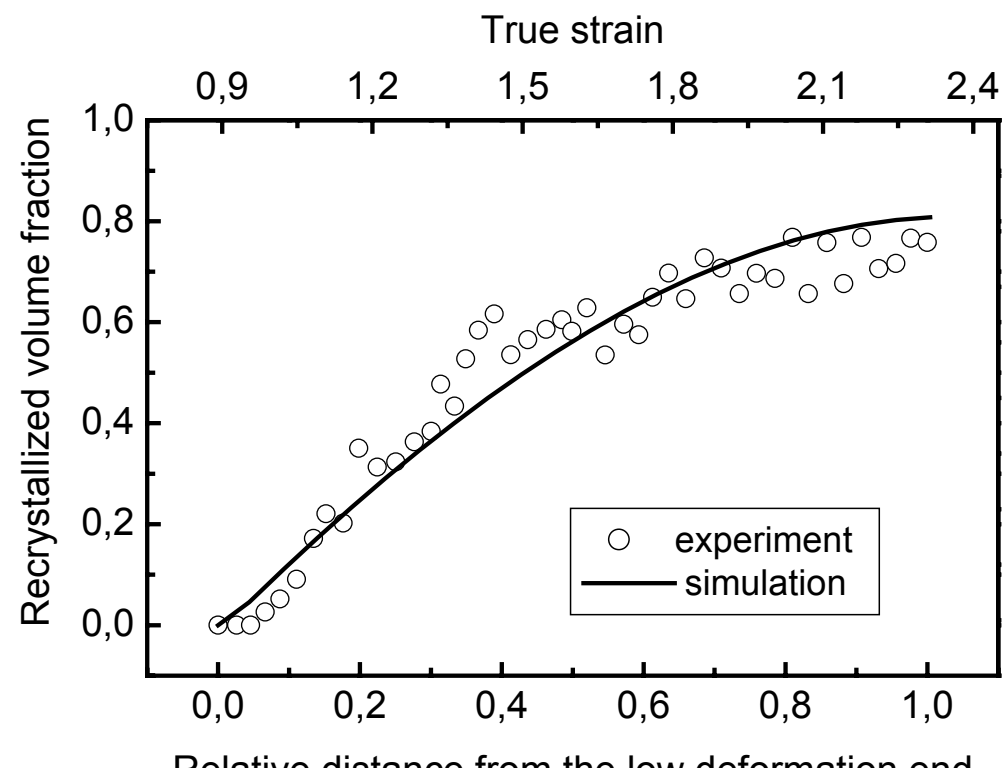

Fig. 6. Variation of the recrystallized volume fraction with position.

\section{CONCLUSIONS}

A 3D Monte Carlo model that correlates the stored energy, nucleus density and migration velocity of recrystallizing grain boundaries, to the local deformation, has been developed to simulate the recrystallization behavior in a material containing particles. The evolving microstructures were displayed both in three-dimensions and at different sections, guaranteeing an accurate description of characteristics of the inhomogeneous microstructures. The simulation results agree well both morphologically and quantitatively with experiments of an Al$0.5 \mathrm{wt} . \% \mathrm{Zr}$ alloy containing $\mathrm{Al}_{3} \mathrm{Zr}$ precipitates that has been subjected to graded deformation and annealing. In the case of large scale inhomogeneities in the microstructure, the technique of 3D computer visualization shows advantages for sampling, detection and measuring.

\section{REFERENCES}

Doherty RD, Hughes DA, Humphreys FJ, Jonas JJ, Juul Jensen D, Kassner ME, et al. (1997). Current issues in recrystallization: a review. Mater Sci Eng A238:219-74. 
Enomoto Y, Kato R (1990). Scaling behavior of twodimensional vertex model for normal grain growth. Acta Metall Mater 38:765-9.

Exner HE, Hougardy HP (1988). Quantitative image analysis of microstructures. Oberursel, Germany: DGM Informationsgesellschaft.

Krill III CE, Chen LQ (2002). Computer simulation of 3-D grain growth using a phase-field model. Acta Mater 50:3057-73.

Raabe D (2002). Cellular Automata in materials science with particular reference to recrystallization simulation. Annu Rev Mater Res 32:53-76.

Rollett AD, Srolovitz DJ, Anderson MP, Doherty RD (1992).
Computer simulation of recrystallization. Acta Metall Mater 40:3475-95.

Song X, Liu G (1998). A simple and efficient threedimensional Monte Carlo simulation of grain growth. Scripta Mater 38:1691-6.

Song X, Rettenmayr M, Müller C, Exner HE (2001). Modeling of recrystallization after inhomogeneous deformation. Metall Mater Trans 32A:2199-206.

Srolovitz DJ, Anderson MP, Grest GS (1983). Grain growth in two dimensions. Scripta Metall 17:241-6.

Yu Y, Liu G (1989). Stereology-principles and applications of quantitative analysis of microstructures. Beijing, China: Metallurgical Industry press. 